\title{
WEAKLY PRIMAL GRADED SUPERIDEALS
}

\author{
AMEER JABER
}

\begin{abstract}
Let $G$ be an abelian group and let $R$ be a commutative $G$-graded super-ring (briefly, graded super-ring) with unity $1 \neq 0$. We say that $a \in h(R)$, where $h(R)$ is the set of homogeneous elements in $R$, is weakly prime to a graded superideal $I$ of $R$ if $0 \neq r a \in I$, where $r \in h(R)$, then $r \in I$. If $v(I)$ is the set of homogeneous elements in $R$ that are not weakly prime to $I$, then we define $I$ to be weakly primal if $P=\bigoplus_{g \in G}\left(v(I) \cap R_{g}^{0}+v(I) \cap\right.$ $\left.R_{g}^{1}\right) \cup\{0\}$ forms a graded superideal of $R$. In this paper we study weakly primal graded superideals of $R$. Moreover, we classify the relationship among the families of weakly prime graded superideals, primal and weakly primal graded superideals of $R$.
\end{abstract}

\section{Introduction}

Weakly prime ideals in commutative rings have been introduced and studied by $\mathrm{D}$. D. Anderson and E. Smith in [1], weakly prime graded ideals in commutative graded rings have been studied by many authors such as S. E. Atani in [3], and primal ideals in commutative rings have been introduced by Ladislas Fuchs in [6]. Graded super-rings are new important concept. They have been studied by many authors, includes Y. A. Bahturin in [4]. Because of the importance of the graded super-rings, we generalize the concept of primal, weakly primal and weakly prime to the graded supercase. Therefore, we introduce and study primal, weakly primal graded superideals and weakly prime graded superideals. Moreover, we classify the relationship among the families of weakly prime graded superideals, primal and weakly primal graded superideals. A number of results and examples concerning weakly primal graded superideals are given.

Let $G$ be an abelian group and let $R$ be any commutative ring with unity, then $R$ is called a G-graded super-ring (for short graded super-ring), if $R=\bigoplus_{g \in G}\left(R_{g}^{0}+R_{g}^{1}\right)$, such that if $a, b \in G$, then $R_{a}^{\alpha} R_{b}^{\beta} \subseteq R_{a b}^{\alpha+\beta}$, where $\alpha, \beta \in \mathbb{Z}_{2}$. Let $h_{0}(R)=\cup_{g \in G} R_{g}^{0}$ and $h_{1}(R)=\cup_{g \in G} R_{g}^{1}$, then $h(R)=$ $h_{0}(R) \cup h_{1}(R)$ is the set of homogeneous elements in $R$ and $1 \in R_{e}^{0}$, where $e$ is the identity element in $G$.

2010 Mathematics Subject Classification. 13A02, 16D25, 16W50.

Key words and phrases. Prime graded superideal, weakly prime graded superideal, primal graded superideal, weakly primal graded superideal. 
We say that an element $a \in h(R)$ is weakly prime to a graded superideal $I$ of $R$ if $0 \neq r a \in$ $I$, where $r \in h(R)$, then $r \in I$. If $I$ is a proper graded superideal of $R$ and $v(I)$ is the set of homogeneous elements of $R$ that are not weakly prime to $I$, then we define $I$ to be weakly primal if the set $P=\bigoplus_{g \in G}\left(v(I) \cap R_{g}^{0}+v(I) \cap R_{g}^{1}\right) \cup\{0\}$ forms a graded superideal of $R$. In this case we say that $I$ is a $P$-weakly primal graded superideal of $R$. By the definition of weakly primal graded superideal we have that $I=\{0\}$ is a $\{0\}$-weakly primal graded superideal of $R$. Moreover, it is easy to see that if $I$ is a $P$-weakly primal graded superideal of $R$, then $v(I)=$ $h(P)-\{0\}$.

We define a proper graded superideal $P$ of $R$ to be weakly prime (prime) if $0 \neq a b \in P$ $(a b \in P$ ) implies that $a \in P$ or $b \in P$, where $a, b \in h(R)$. Let $I$ be a proper graded superideal of $R$, an element $a \in h(R)$ is called prime to $I$ if $a r \in I$, where $r \in h(R)$, then $r \in I$. If $I$ is a proper graded superideal of $R$ and $\rho(I)$ is the set of homogeneous elements of $R$ that are not prime to $I$, then we define $I$ to be primal if the set $P=\bigoplus_{g \in G}\left(\rho(I) \cap R_{g}^{0}+\rho(I) \cap R_{g}^{1}\right)$ forms a graded superideal of $R$. In this case we say that $I$ is a $P$-primal graded superideal of $R$. Moreover, if $I$ is a $P$-primal graded superideal of $R$, then it is easy to check that $P$ is a prime graded superideal of $R$.

A graded superideal $I$ of $R$ is said to be irreducible if whenever $I$ is the intersection of two graded superideals of $R$ then $I$ is one of them.

If $I$ and $J$ are graded superideals of $R$, then the graded superideal $\{r \in R: r J \subseteq I\}$ is denoted by $(I: J)$. A homogeneous element in $R$ is regular if it is a nonzero divisor in $R$. That is $a \in h(R)$ is regular if $(0: a)=0$. A commutative graded super-ring $R$ with unity is called a graded superdomain if $h(R)$ has no divisors of zero.

In [2], S. E. Atani defined the prime subgroup $P_{g}$ of $R_{g}$ for $g \in G$, where $P$ is a graded ideal in $R$. Throughout this work we always assume that if $P$ is a graded superideal (ideal) of $R$, then $P_{e}^{1}$ is not a prime subgroup of $R_{e}^{1}$, and $P_{g}^{\alpha}\left(P_{g}\right)$ is not a prime subgroup of $R_{g}^{\alpha}\left(R_{g}\right)$ for all $g \neq e \in G$ and $\alpha \in \mathbb{Z}_{2}$.

\section{Weakly prime graded superideals}

Let $R$ be a commutative $G$-graded super-ring and let $I$ be a graded superideal of $R$. Then every element prime to $I$ is weakly prime to $I$, but the converse is not true. For example if $R=$ $\mathbb{Z}_{24}+u \mathbb{Z}_{24}$ with $u^{2}=0$, then $R$ is a commutative super-ring with unity. Let $I=8 \mathbb{Z}_{24}+u \mathbb{Z}_{24}$, then $I$ is a superideal of $R$ and $\overline{6}$ is weakly prime to $I$, since if $\bar{a} \in h(R)$, then $\bar{a} \cdot \overline{6} \in I$ if and only if $\bar{a} \cdot \bar{b}=0$ or $\bar{a} \in u \mathbb{Z}_{24} \subseteq I$. But $\overline{6}$ is not prime to $I$, since $\overline{4} \cdot \overline{6} \in I$ and $\overline{4} \notin I$.

Let $R$ be a commutative $G$-graded super-ring which is not a graded superdomain. Then, by definition, $\{0\}$ is a $\{0\}$-weakly primal graded superideal of $R$ but not primal; hence weakly primal graded superideals need not to be primal graded superideals. 
Let $R$ be a $G$-graded super-ring (briefly graded super-ring) with unity. We start this section by giving two characterizations of weakly primal graded superideals of $R$.

Theorem 2.1. Let I and $P$ be proper graded superideals of $R$. Then the following statements are equivalent.

(1) I is a P-weakly primal graded superideal of $R$.

(2) For $x \notin h(P)-\{0\}, h((I: x))=h(I) \cup h((0: x))$ and for $x \neq 0 \in h(P), h(I) \cup h((0: x)) \varsubsetneqq h((I: x))$.

Proof. (1) $\Rightarrow$ (2) Let $I$ be a $P$-weakly primal graded superideal of $R$. Then $v(I)=h(P)-\{0\}$. If $x \notin v(I)$, then $x$ is weakly prime to $I$. Let $r \in h((I: x))$. If $0 \neq r x \in I$, then $r \in I$, since $x$ is weakly prime to $I$. If $r x=0$, then $r \in(0: x)$. Therefore we have $h((I: x)) \subseteq h(I) \cup h((0: x))$ but also we clearly have that $I \subseteq(I: x)$ and $(0: x) \subseteq(I: x)$; hence $h((I: x))=h(I) \cup h((0: x))$.

Now, assume that $x \neq 0 \in h(P)$ (i.e. $x \in v(I)$ ). Then $x$ is not weakly prime to $I$; hence there exists $r \in h(R)-h(I)$ such that $0 \neq r x \in I$. Thus $r \in h((I: x))$, but $r \notin h(I) \cup h((0: x))$. Therefore, $h(I) \cup h((0: x)) \varsubsetneqq h((I: x))$.

(2) $\Rightarrow$ (1) By (2) we have $v(I)=h(P)-\{0\}$. Thus $I$ is a $P$-weakly primal graded superideal of $R$.

We remark that if $A, B, C$ are graded superideals of $R$ with $h(A)=h(B) \cup h(C)$ then $A=$ $B+C$, but the converse need not to be true. For example $2 \mathbb{Z}=4 \mathbb{Z}+10 \mathbb{Z}$ but $2 \notin 4 \mathbb{Z} \cup 10 \mathbb{Z}$.

Lemma 2.2. Let I be a proper graded superideal of R. Then the following hold.

(1) If I is a P-weakly primal graded superideal of $R$, then $I \subseteq P$.

(2) $I$ is a $\{0\}$-weakly primal graded superideal of $R$ if and only if $I=\{0\}$.

Proof. (1) If $0 \neq a \in h(I)$, then $1 . a \in I$ and $1 \notin I$, so $a$ is not weakly prime to $I$. Thus $a \in P$ and hence $I \subseteq P$.

(2) This follows from (1) and the definition of weakly primal graded superideal.

The next two results are very important facts about weakly primal graded superideals, and will be used throughout the rest of the paper.

Theorem 2.3. Every weakly prime graded superideal of $R$ is weakly primal.

Proof. Let $P$ be a weakly prime graded superideal of $R$. Then $P \neq\{0\}$. It suffices to show that $v(P)=h(P)-\{0\}$. Let $0 \neq a \in h(P)$. Then $a=1 . a \in P$ with $1 \notin P$, so $a$ is not weakly prime to $P$ and hence $a \in v(P)$. On the other hand, if $a \notin h(P)-\{0\}$, then because $P$ is weakly prime, we have $h((P: a))=h(P) \cup h((0: a))$, so if $r \in h(R)$ with $0 \neq r a \in P$, then $r \in h(P)$ and hence $a$ is weakly prime to $P$, so $a \notin v(P)$. Thus $v(P)=h(P)-\{0\}$; hence $P$ is a weakly primal graded superideal of $R$. 
Proposition 2.4. If I is a $P$-weakly primal graded superideal of $R$, then $P$ is a weakly prime graded superideal of $R$.

Proof. Suppose that $a, b \in h(R)-h(P)$ we show that $a b=0$ or $a b \notin h(P)$. Assume that $a b \neq 0$ and let $0 \neq r a b \in I$ for some $r \in h(R)$. Then by Theorem 2.1 we have $0 \neq r a \in h((I: b))=$ $h(I) \cup h((0: b))$, but $r a \notin h((0: b))$; hence $0 \neq r a \in h(I)$. Then, from Theorem $2.1,0 \neq r \in h((I:$ $a))=h(I) \cup h((0: a))$, where $r a \neq 0$. So, $r \in h(I)$ and hence $a b$ is weakly prime to $I$. Thus $a b \notin h(P)$.

Now we give an example of a weakly primal superideal of $R$ that is not weakly prime.

Example 2.5. Let $R=\mathbb{Z}_{8}+u \mathbb{Z}_{8}$ where $u^{2}=0$. Then $R$ is a commutative super-ring with unity. If $I=4 \mathbb{Z}_{8}+u \mathbb{Z}_{8}$ then $I$ is not a weakly prime superideal of $R$, since $\overline{2} \cdot \overline{2} \neq 0 \in I$, but $\overline{2} \notin I$. Let $P=2 \mathbb{Z}_{8}+u \mathbb{Z}_{8}$, we show that $I$ is a $P$-weakly primal superideal of $R$. It is enough to show that $v(I)=h(P)-\{0\}$. Let $0 \neq \bar{a} \in h(P)$, if $\bar{a} \in 2 \mathbb{Z}_{8}$ then $\bar{a}=2 k \in \mathbb{Z}_{8}$. If $k$ is an odd number, then $0 \neq \overline{2} \bar{a} \in I$, but $\overline{2} \notin I$, and if $k$ is an even number $0 \neq \overline{1} \bar{a} \in I$ with $\overline{1} \notin I$; hence $\bar{a} \in v(I)$. If $\bar{a} \in u \mathbb{Z}_{8}$ then $\bar{a} \in I \subseteq v(I)$. On the other hand, if $\bar{a} \in h(R)-h(P)$ then $\bar{a}$ is an odd number in $\mathbb{Z}_{8}$. If $0 \neq \bar{a} \bar{m} \in I$ for some $\bar{m} \in \mathbb{Z}_{8}$ then 4 divides $a m$ and so, 4 divides $m$ since $(4, a)=1$; hence $\bar{m} \in I$. Thus $I$ is a $P$-weakly primal superideal of $R$.

The following corollary depends on the fact if $(R, P)$ is a quasilocal graded super-ring with $P^{2}=\{0\}$, then every nontrivial proper graded superideal of $R$ is a weakly prime graded superideal.

Corollary 2.6. If $(R, P)$ is a quasilocal graded super-ring with $P^{2}=\{0\}$, then every nontrivial proper graded superideal of $R$ is a weakly primal graded superideal.

We recall that if $P$ is a weakly prime graded superideal in $R$, and $P_{e}^{0}$ is not a prime ideal in $R_{e}^{0}$, where $e$ is the identity element in $G$, then $\left(P_{e}^{0}\right)^{2}=\{0\}$ (See [1, Theorem 3]). Next we have the following results about graded superideals.

Theorem 2.7. Let $P$ be a graded superideal in $R$, where $R$ is a graded super-ring and where $P_{e}^{0}$ is not a prime ideal in $R_{e}^{0}$. Then $\left(P_{g}\right)^{4}=\{0\}$ for all $g \in G$.

Proof. Let $a=a^{0}+a^{1} \in P_{g}$. Then $a^{2}=\left(a^{0}\right)^{2}+\left(a^{1}\right)^{2}+2 a^{0} a^{1}=2 a^{0} a^{1}$ which implies that $a^{4}=$ $4\left(a^{0}\right)^{2}\left(a^{1}\right)^{2}=0$. Thus $\left(P_{g}\right)^{4}=\{0\}$.

The next result on weakly prime graded superideals depends on Theorem 2.7.

Theorem 2.8. Assume that $G$ is a finite abelian group of order $n \geq 2$. If $P$ is a graded superideal of $R$, where $P_{e}^{0}$ is not a prime ideal in $R_{e}^{0}$. Then $P^{4 n}=\{0\}$. 
Proof. Let $c=a \in P_{g}$ then, by Theorem 2.7, $c^{4}=0$. Suppose for $c=a_{1}+\cdots+a_{k} \in P$, where $a_{i} \in P_{g_{i}}$ for all $i=1,2, \ldots, k$ and $k \leq n-1, c^{4 k}=0$. We show that it is true for $n$. Let $c=$ $a_{1}+\cdots+a_{n} \in P$, where $a_{i} \in P_{g_{i}}$ for all $i$, then $c=a_{1}+x$ where $x=a_{2}+\cdots+a_{n}$ and

$$
c^{4(n-1)}=m_{4 n-5} a_{1}^{4 n-5} x+\cdots+m_{1} a_{1} x^{4 n-5}=m_{3} a_{1}^{3} x^{4 n-7}+m_{2} a_{1}^{2} x^{4 n-6}+m_{1} a_{1} x^{4 n-5}
$$

where $m_{i} \in \mathbb{Z}^{+}$; hence

$$
c^{4 n}=c^{4(n-1)} c^{4}=\left(m_{3} a_{1}^{3} x^{4 n-7}+m_{2} a_{1}^{2} x^{4 n-6}+m_{1} a_{1} x^{4 n-5}\right)\left(m^{\prime} a_{1} x^{3}+m^{\prime \prime} x^{4}\right)=0,
$$

since $a_{1}^{4}=0$ or $x^{4(n-1)}=0$. Thus, $c^{4 n}=0$ which implies that $P^{4 n}=\{0\}$.

Easy computations give us the following corollary of Theorem 2.8.

Corollary 2.9. Let $P$ be a weakly prime graded superideal of $R$, where $R$ is a graded super-ring, and where $P_{e}^{0}$ is not a prime ideal in $R_{e}^{0}$. Then $P^{4 n-1} \sqrt{0}=\{0\}$.

Now we have the following known result in the ungraded case.

Proposition 2.10. Let $R$ be a commutative ring with unity. If $P$ and $Q$ are weakly prime ideals of $R$, but not prime ideals then $P Q=\{0\}$.

Proof. See [1, Corollary 5].

Following the same proof used in Theorem 2.8 we have the following result about graded ideals.

\section{Corollary 2.11.}

(1) Let $P$ be a weakly prime graded ideal of $R$, where $P_{e}$ is not a prime ideal in $R_{e}$, and let $|G|=n$, then $P^{2 n}=\{0\}$.

(2) If $|G|=1$, then $P^{2}=\{0\}$.

Depending on Theorem 2.7, [1, Theorem 3], Proposition 2.10 and [2, Theorem 2.6], we have the following remark, where $P$ and $Q$ are weakly prime graded superideals (ideals) of $R$ and where $P_{e}^{0}\left(P_{e}\right)$ and $Q_{e}^{0}\left(Q_{e}\right)$ are not prime ideals in $R_{e}^{0}\left(R_{e}\right)$.

\section{Remark 2.12.}

(1) Let $P$ and $Q$ be weakly prime graded ideals of $R$. Then by [2, Theorem 2.6], $P+Q$ is a weakly prime graded ideal of $R$.

(a) If $\left(P_{g}+Q_{g}\right)^{2}=\{0\}$, then $P_{g} Q_{g}=\{0\}$ for all $g \neq e \in G$.

(b) $\left(P_{g} Q_{h}\right)^{2}=\{0\}$ for all $g, h \in G$. 
(c) If $P_{e}+Q_{e}$ is not a prime ideal in $R_{e}$, then $\left(P_{e}+Q_{e}\right)^{2}=\{0\}$; hence $P_{e} Q_{e}=\{0\}$.

(2) Let $P$ and $Q$ be weakly prime graded superideals of $R$. Then by [2, Theorem 2.6], $P+Q$ is a weakly prime graded superideal of $R$.

(a) If $\left(P_{g}+Q_{g}\right)^{4}=\{0\}$ for all $g \neq e \in G$, then $P_{g} Q_{g}\left(P_{g}+Q_{g}\right)^{2}=\{0\}$.

(b) $\left(P_{g} Q_{h}\right)^{4}=\{0\}$ for all $g, h \in G$.

(c) If $\left(P_{e}^{1}+Q_{e}^{1}\right)^{2}=\{0\}$ and $\left(P_{e}^{0}+Q_{e}^{0}\right)$ is not a prime ideal in $R_{e}^{0}$, then $\left(P_{e}+Q_{e}\right)^{4}=\{0\}$.

Now depending on [1, Theorem 3], Theorem 2.8 and Corollary 2.9 we have the following result.

Theorem 2.13. Let I be a $P$-weakly primal graded superideal of $R$ and $J$ a $Q$-weakly primal graded superideal of $R$, where $P_{e}^{0}$ and $Q_{e}^{0}$ are not prime ideals in $R_{e}^{0}$ and where $|G|=n$. Then the following statements are hold.

(1) $I^{4 n}=\{0\}, I P^{4 n-1}=\{0\}, I^{4 n-1} \sqrt{0}=\{0\}$ and $\sqrt{I}=\sqrt{\{0\}}$.

(2) $(I J)^{4 n}=\{0\}$.

Proof. (1) By Lemma 2.2, Proposition 2.4, [1, Theorem 3] and Theorem 2.8 we get $I^{4 n} \subseteq P^{4 n}=$ $\{0\}, I P^{4 n-1} \subseteq P^{4 n}=\{0\}$ and $I^{4 n-1} \sqrt{0} \subseteq P^{4 n-1} \sqrt{0}=\{0\}$. Finally, since $I^{4 n}=\{0\}$, we get $I \subseteq \sqrt{0}$; hence $\sqrt{I} \subseteq \sqrt{0}$, but $\sqrt{0} \subseteq \sqrt{I}$ is trivial, so $\sqrt{I}=\sqrt{\{0\}}$.

(2) By Lemma 2.2, Proposition 2.4, [1, Theorem 3] and Theorem 2.8 we have $(I J)^{4 n} \subseteq$ $P^{4 n} Q^{4 n}=\{0\}$. Thus, $(I J)^{4 n}=\{0\}$.

In Theorem 2.8 and Corollary 2.11 we showed that if $P$ is a weakly prime graded superideal (ideal) of $R$, where $P_{e}^{0}\left(P_{e}\right)$ is not a prime ideal in $R_{e}^{0}\left(R_{e}\right)$, then $P^{4 n}=\{0\}\left(P^{2 n}=\{0\}\right)$, where $n=|G|$. In the next result we will use an important fact for finite abelian groups to improve Theorem 2.8 and Corollary 2.11. Compare the next result with Theorem 2.8 and Corollary 2.11 .

\section{Theorem 2.14.}

(1) Let $|G|=2 k$ or $2 k+1$, and let $P$ be weakly prime graded ideal of $R$ where $P_{e}$ is not a prime ideal in $R_{e}$. Then $P^{k+2}=\{0\}$.

(2) Let $|G|=2 k$ or $2 k+1$, and let $P$ be a weakly prime graded superideal of $R$ where $P_{e}^{0}$ is not a prime ideal in $R_{e}^{0}$. Then $P^{|G|+2}=\{0\}$.

Proof. If $|G|=1$ or $|G|=2$ and $P$ is a weakly prime graded ideal of $R$ where $P_{e}$ is not a prime ideal in $R_{e}$ then by [1, Theorem 3], $\left(P_{e}\right)^{2}=\{0\}$. This implies that $P^{2}=\{0\}$ if $|G|=1$, or $P^{3}=\{0\}$ if $|G|=2$. Similarly, if $|G|=1$ or $|G|=2$ and $P$ is a weakly prime graded superideal of $R$ where 
$P_{e}^{0}$ is not a prime ideal in $R_{e}^{0}$ then by [1, Theorem 3], $\left(P_{e}^{0}\right)^{2}=\{0\}$. This implies that $P^{3}=\{0\}$ if $|G|=1$, or $P^{4}=\{0\}$ if $|G|=2$.

Next suppose that $G$ is a finite abelian group of order $2 k$ or $2 k+1$ 嫩where $k \geq 2$. We prove (1) and (2) by using the following fact about $G$. Let $S \subseteq G$ with $|S|=k+2$. For any $s \neq e \in S$, let $T=S-\{s\}$. Then $T \cap s T \neq \varnothing$. Because, if $T \cap s T=\varnothing$ then $T \cup s T \subseteq G$ and $|T \cup s T|=2(k+1)=2 k+2>|G|$, a contradiction. Therefore, $T \cap s T \neq \varnothing$. Let $x \in T \cap s T$, then there exists $y \in T$ with $s y=x$. Since $s \neq e$, we have $x \neq y$. Moreover, $x \neq s$ and $y \neq s$, since $x, y \in T=S-\{s\}$.

(1) Now, since $P_{e}$ is weakly prime ideal in $R_{e}$ but not a prime ideal we have that $\left(P_{e}\right)^{2}=\{0\}$. So, to show that $P^{k+2}=\{0\}$, then it is enough to prove that for any $p_{1}, p_{2}, \ldots, p_{k+2} \in h(P)$ we have $p_{1} . p_{2} \ldots . . p_{k+2}=0$. Let $p_{1}, p_{2}, \ldots, p_{k+2} \in P$, where each $p_{i} \in P_{g_{i}}$ for all $i=1,2, \ldots, k+2$ and $g_{i} \neq g_{j}$ for all $i \neq j$. If $g_{i}=g_{j}$ for some $i, j$ between 1 and $k+2$, then $p_{1}$. $p_{2} \ldots . . p_{k+2} \in$ $\left(P_{g_{i}}\right)^{2}=\{0\}$. Therefore we may assume that $g_{i} \neq g_{j}$ for all $i \neq j$. Let $g_{i} \neq e$ for some $i$ between 1 and $k+2$ then by the fact above if $T=\left\{g_{1}, g_{2}, \ldots, g_{k+2}\right\}-\left\{g_{i}\right\}$ then there exist $n \neq m$ between 1 and $k+2$ such that $g_{n}, g_{m} \in T$ and $g_{i}=g_{n} g_{m}$. Thus $p_{1}, p_{2}, \ldots, p_{k+2} \in$ $\left(P_{g_{i}}\right)^{2}=\{0\}$; hence $P^{k+2}=\{0\}$.

(2) Since $P_{e}^{0}$ is not a prime ideal in $R_{e}^{0}$ we have $\left(P_{e}^{0}\right)^{2}=\{0\}$.

In part (2), we may assume that $P$ is a weakly prime graded ideal of $R$ over the new abelian group $G^{\prime}=\mathbb{Z}_{2} \times G$. So, by using part (1) we get that $P^{m}=\{0\}$ for any $m \geq|G|+2$.

We end this section by the following result. Compare the next result with Proposition 2.10.

Theorem 2.15. Let $P$ and $Q$ be weakly prime graded superideals (ideals) of $R$, where $|G|=n$, and let $P_{e}^{0}\left(P_{e}\right)$ and $Q_{e}^{0}\left(Q_{e}\right)$ be not prime ideals in $R_{e}^{0}\left(R_{e}\right)$.

(1) If $P$ and $Q$ are weakly prime graded ideals of $R$, then $(P Q)^{2}=\{0\}$.

(2) If $P$ and $Q$ be weakly prime graded superideals of $R$, then $(P Q)^{2}=\{0\}$.

Proof. (1) We prove it by induction on $k \leq n$. Let $k=1$ and let $a=a_{1} \in h(P Q)$, then $a=p_{g} q_{h}$, where $p_{g} \in P_{g}$ and $q_{h} \in Q_{h}$. Then $a^{2}=p_{g}^{2} q_{h}^{2}=0$. Suppose $a^{2}=0$ for any $a=a_{1}+\cdots+a_{k}$, where $k \leq n-1$ and $a_{i} \in h(P Q)$ for all $i=1, \ldots, k$. Now, let $a=a_{1}+a_{2} \cdots+a_{n}$, where $a_{i} \in h(P Q)$ for all $i=1, \ldots, n$. Then $a=a_{1}+x$, where $x=a_{2}+\cdots+a_{n}$, and by induction hypothesis $a^{2}=$ $a_{1}^{2}+2 a_{1} x+x^{2}=0+2 a_{1} x+0$. Thus, $a^{2}=2 a_{1} x$. Now, $a_{1} x=a_{1} a_{2}+\cdots+a_{1} a_{n}$. For any $j=2,3, \ldots, n$ then by induction hypothesis we have that $\left(a_{1}+a_{j}\right)^{2}=0$, so $\left(a_{1}+a_{j}\right)^{2}=0=a_{1}^{2}+a_{j}^{2}+2 a_{1} a_{j}$. Since $a_{1}^{2}=a_{j}^{2}=0$ we get that $2 a_{1} a_{j}=0$; hence $a^{2}=2 a_{1} x=0$. Therefore, $(P Q)^{2}=\{0\}$.

(2) The proof is similar to that of (1). 


\section{Weakly primal graded superideals}

We start this section by recalling that there is a one-to-one correspondence between weakly prime graded superideals of $R$ containing the weakly prime graded superideal $J$ of $R$ and weakly prime graded superideals of $R / J$. Therefore, we have the following result.

Theorem 3.1. Let $I$ be a proper graded superideal of $R$, and let $J$ be a weakly prime graded superideal of $R$ with $J \subseteq I$. Then I is a weakly primal graded superideal of $R$ if and only if $I / J$ is a weakly primal graded superideal of $R / J$.

Proof. Suppose that $I$ is a $P$-weakly primal graded superideal of $R$ with $J \subseteq I$. Then by Lemma 2.2(1) and Proposition 2.4, $P$ is a weakly prime graded superideal of $R$ containing $J$. Therefore, $P / J$ is a weakly prime graded superideal of $R / J$. It suffices to show that $v(I / J)=$ $h(P / J)-\{0\}$. Let $0 \neq a+J \in h(P / J)$. Then $0 \neq a$ is not weakly prime to $I$; hence there exists $r \in h(R)-(I)$ such that $0 \neq r a \in I$. If $r a \in J$ then, because $J$ is a weakly prime graded superideal of $R$ and $a \notin J, r \in J$ which is a contradiction since $J \subseteq I$ and $r \notin I$. It follows that $0 \neq(r+J)(a+J) \in I / J$ with $r+J \notin h(I / J)$, therefore, $a+J$ is not weakly prime to $I / J$. Now assume that $b+J \in h(R / J)$ is not weakly prime to $I / J$. Then there exists $c+J \in h(R / J)-(I / J)$ such that $0 \neq c b+J \in I / J$; hence $0 \neq c b \in I$ with $c \in h(R)-h(I)$. So, $b \neq 0$ is not weakly prime to $I$; hence $0 \neq b \in h(P)$ and therefore, $b+J \in h(P / J)-\{0\}$. Thus, $v(I / J)=h(P / J)-\{0\}$.

Conversely, suppose that $I / J$ is a $P / J$-weakly primal graded superideal of $R / J$; we show that $I$ is a $P$-weakly primal graded superideal of $R$. By Proposition $2.4, P / J$ is a weakly prime graded superideal of $R / J$ and hence $P$ is a weakly prime graded superideal of $R$ containing $J$. It is enough to show that $v(I)=h(P)-\{0\}$. Let $0 \neq a \in h(P)$. By Lemma 2.2(1), we can assume that $a \notin I$. Therefore, $0 \neq a+J \in P / J$. As $I / J$ is a $P / J$-weakly primal graded superideal of $R / J$, there exists $r+J \in h(R / J)-h(I / J)$ such that $0 \neq r a+J \in I / J$; hence $0 \neq r a \in I$ with $r \in h(R)-h(I)$. Thus $a$ is not weakly prime to $I$ that is $a \in v(I)$. Now assume that $a \in v(I)$. So, $a \neq 0$. If $a \in h(I)$ then $a \in h(P)$, so we can assume that $a \notin h(I)$. Then there is an element $r \in h(R)-h(I)$ with $0 \neq r a \in I$. Because $J$ is a weakly prime graded superideal of $R$ and $a \notin h(J)$ and $r \notin J$ we get that $r a \notin J$. Therefore, $0 \neq r a+J=(r+J)(a+J) \in I / J$ with $r+J \in h(R / J)-h(I / J)$; hence $a+J \in h(P / J)-\{0\}$, since $I / J$ is a $P / J$-weakly primal graded superideal of $R / J$. Therefore, $0 \neq a \in h(P)$ which implies that $v(I)=h(P)-\{0\}$.

Let $S$ be a subset of $h(R)-\{0\}$, where $R$ is a graded super-ring. Then $S$ is called a multiplicatively closed subset of $R$ if for any $a, b \in S$ we have $a b \in S$.

Lemma 3.2. Let $S$ be a multiplicatively closed subset of $R$. Then the following hold.

(1) If I is a $P$-weakly primal graded superideal of $R$ with $h(P) \cap S=\varnothing$ and $0 \neq a / s \in S^{-1} I$, then $a \in I$. 
(2) If $Q$ is a weakly prime graded superideal of $R$ with $h(Q) \cap S=\varnothing$ and $0 \neq a / s \in S^{-1} Q$, then $a \in Q$.

Proof. First assume that $0 \neq a / s \in S^{-1} I$ but $a \in h(R)-h(I)$. Then $a / s=r / t$ for $r \in h(I)$ and $t \in S$, so there exists $u \in S$ such that $0 \neq u t a=u s r \in I$ with $a \notin I$. Hence $u t \in S$ is not weakly prime to $I$ which is a contradiction. Thus, $a \in h(I)$.

(2) This follows from (1) and Theorem 2.3.

Recall that $a \in h(R)$ is a regular element in $R$, if $a$ is a nonzero divisor; that is $a \in h(R)$ is a regular element in $R$, if $(0: a)=\{0\}$. In the rest of this section, we always assume that if $S$ is a multiplicatively closed subset of $R$, then $S$ consists of regular elements.

Proposition 3.3. Let $S$ be a multiplicatively closed subset of $R$ and let I be a P-weakly graded primal superideal of $R$ such that $h(P) \cap S=\varnothing$. Then the following hold.

(1) $S^{-1} I$ is a $S^{-1} P$-weakly primal graded superideal of $S^{-1} R$.

(2) $I=S^{-1} I \cap R$.

Proof. (1) We will show that $v\left(S^{-1} I\right)=h\left(S^{-1} P\right)-\{0\}$. Let $0 \neq \frac{a}{s} \in h\left(S^{-1} P\right)$. Then, by Lemma 3.2(2), $0 \neq a \in h(P)$; hence there exists $r \in h(R)-h(I)$ such that $0 \neq r a \in I$. Because $S$ consists of regular elements in $R$, we have $\frac{r a}{s} \neq 0 \in S^{-1} I$. Thus, $0 \neq \frac{r a}{s}=\left(\frac{r}{1}\right)\left(\frac{a}{s}\right) \in S^{-1} I$ where, by Lemma 3.2(1), $\frac{r}{1} \notin S^{-1} I$. Hence $\frac{a}{s} \in v\left(S^{-1} I\right)$. On the other hand, suppose that $\frac{a}{s} \in v\left(S^{-1} I\right)$. Then there exists $\frac{r}{t} \in h\left(S^{-1} R\right)-h\left(S^{-1} I\right)$ such that $0 \neq\left(\frac{r}{t}\right)\left(\frac{a}{s}\right) \in S^{-1} I$. Because $S$ consists of regular elements in $R, r a \neq 0 \in I$ with $r \notin h(I)$; hence $0 \neq a \in h(P)$. Thus, $\frac{a}{s} \in h\left(S^{-1} P\right)-\{0\}$.

(2) Since it is clear that $I \subseteq S^{-1} I \cap R$; we will prove the reverse inclusion. Let $a \in S^{-1} I \cap$ $h(R)$. Then, $\frac{a}{1} \in S^{-1} I$, so, $a \in h(I)$ by Lemma 3.2(1).

Proposition 3.4. Let $S$ be a multiplicatively closed subset of $R$ and let $Q$ be a weakly graded prime superideal of $S^{-1} R$. Then $Q \cap R$ is a weakly prime graded superideal of $R$.

Proof. Suppose that $0 \neq a b \in Q \cap R$. Then $0 \neq \frac{a b}{1} \in Q$, Since $S$ consists of regular elements, $0 \neq\left(\frac{a}{1}\right)\left(\frac{b}{1}\right) \in Q$. Since $Q$ is weakly prime, we have $\frac{a}{1} \in Q$ or $\frac{b}{1} \in Q$; hence $a \in Q \cap R$ or $b \in Q \cap R$.

Proposition 3.5. Let $S$ be a multiplicatively closed subset of $R$. If I is a $Q$-weakly primal graded superideal of $S^{-1} R$, then $I \cap R$ is a $Q \cap R$-weakly primal graded superideal of $R$.

Proof. By Proposition 3.4, $P=Q \cap R$ is a weakly prime graded superideal of $R$. We will show that $h(P)-\{0\}=v(I \cap R)$. Suppose that $0 \neq a \in h(P)$. Then $0 \neq \frac{a}{1} \in Q$, so there exists $\frac{r}{s} \in$ 
$h\left(S^{-1} R\right)-h(I)$ such that $0 \neq\left(\frac{r}{s}\right)\left(\frac{a}{1}\right) \in I$. Hence $0 \neq r a \in I \cap R$ with $r \in h(R)-h(I \cap R)$. It follows that $a \in v(I \cap R)$. On the other hand assume that $b \in v(I \cap R)$, where $b \neq 0$. Then there is an element $s \in h(R)-h(I \cap R)$ with $0 \neq s b \in I \cap R$, where $\frac{s b}{1} \neq 0 \in I$ since $S$ consists of regular elements. So, $\left(\frac{s}{1}\right)\left(\frac{b}{1}\right) \neq 0 \in I$ with $\frac{s}{1} \notin I$ implies that $\frac{b}{1} \in Q$. Thus, $b \in h(P)$.

Let $P$ be a weakly prime graded superideal of $R$ with $h(P) \cap S=\varnothing$, where $S$ is a multiplicatively closed subset of $R$ consists of regular elements. Then $S^{-1} P$ is a weakly prime graded superideal of $S^{-1} R$. On the other hand if $N$ is a proper weakly prime graded superideal of $S^{-1} R$, then $P=N \cap R$ is a weakly prime graded superideal of $R$ with $h(P) \cap S=\varnothing$, since if $a \in h(P) \cap S$ then $\frac{a}{1} \in N$ which implies that $1 \in N$; hence $N=S^{-1} R$, a contradiction. Therefore, we can say that there is a one-to-one correspondence between weakly prime graded superideals $P$ of $R$ with $h(P) \cap S=\varnothing$ and weakly prime graded superideals of $S^{-1} R$, where $S$ is a multiplicatively closed subset of $R$ consists of regular elements. Now, using this fact, we are able to prove the following result.

Theorem 3.6. Let $S$ be a multiplicatively closed subset of $R$, and let $P$ be a weakly prime graded superideal of $R$ with $h(P) \cap S=\varnothing$. Then there exists a one-to-one correspondence between the $P$-weakly primal graded superideals of $R$ and the $S^{-1} P$-weakly primal graded superideals of $S^{-1} R$.

Proof. This follows from Propositions 3.3, 3.4, 3.5 and the fact that there is a one-toone correspondence between weakly prime graded superideals of $R$ and weakly prime graded superideals of $S^{-1} R$, where $S$ is a multiplicatively closed subset of $R$ consists of regular elements.

\section{Primal graded superideals}

Let $R$ be a commutative graded super-ring which is not a graded superdomain. We recall that 0 is a 0 -weakly primal graded superideal of $R$, but it is not primal. Now, in this section we investigate when a weakly primal graded superideal of a commutative graded super-ring is primal. But first we start by the following example which shows that a primal graded superideal of $R$ need not to be weakly primal.

Example 4.1. Let $R=\left(\mathbb{Z}_{24}+u \mathbb{Z}_{24}\right)+v\left(\mathbb{Z}_{24}+u \mathbb{Z}_{24}\right)$ where $u v=v u$ and $u^{2}=v^{2}=0$. Then $R$ is a commutative $\mathbb{Z}_{2}$-graded super-ring. Let $I=8 \mathbb{Z}_{24}+u \mathbb{Z}_{24}+v\left(\mathbb{Z}_{24}+u \mathbb{Z}_{24}\right)$.

(1) Since $0 \neq \overline{2} \cdot \overline{4} \in I$ with $\overline{2}, \overline{4} \notin I$, then we get that $\overline{2}$ and $\overline{4}$ are not weakly prime to $I$. Easy computations imply that $\overline{2}+\overline{4}=\overline{6}$ is weakly prime to $I$. Thus we obtain that $I$ is not a weakly primal $\mathbb{Z}_{2}$-graded superideal of $R$. 
(2) Set $P=2 \mathbb{Z}_{24}+u \mathbb{Z}_{24}+v\left(\mathbb{Z}_{24}+u \mathbb{Z}_{24}\right)$. We show that $I$ is a $P$-primal $\mathbb{Z}_{2}$-graded superideal of $R$. It is easy to check that every element of $h(P)$ is not prime to $I$. Conversely, assume that $\bar{a} \in h(R)-h(P)$, then $\bar{a} \in \mathbb{Z}_{24}$ with $(a, 8)=1$. If $\bar{a} \cdot \bar{n} \in I$ for some $\bar{n} \in \mathbb{Z}_{24}$ then 8 divides $n$; hence $\bar{n} \in I$. Therefore, $h(P)$ is exactly the set of elements in $h(R)$ which are not prime to $I$. Thus $I$ is a $P$-primal $\mathbb{Z}_{2}$-graded superideal of $R$.

Proposition 4.2. Let $R$ be a commutative super-ring and let I be a $P$-weakly primal superideal of $R$ with $I_{0} I_{\alpha} \neq\{0\}$ for each $\alpha \in \mathbb{Z}_{2}$. If $P$ is a prime superideal of $R$ then $I$ is $P$-primal.

Proof. It is enough to show that $\rho(I)=h(P)$. If $a \in h(P)$, then $a$ is not prime to $I$. So, $a \in \rho(I)$. Assume that $a \in \rho(I)$ we show that $a \in h(P)$. Let $r_{\alpha} \in R_{\alpha}-I_{\alpha}$ such that $r_{\alpha} a \in I$. If $0 \neq r_{\alpha} a \in I$, then $a \in h(P)$ since $I$ is a $P$-weakly primal superideal of $R$. So we may assume that $r_{\alpha} a=0$.

Suppose $a \in R_{\beta}$.

If $a I_{\alpha} \neq\{0\}$, then there exists $s \in I_{\alpha}$ with $a s \neq 0$. So, $r_{\alpha}+s \notin I_{\alpha}$ and $0 \neq a\left(r_{\alpha}+s\right)=a s \in I$. Hence $a \in h(P)$. Therefore, we may assume that $a I_{\alpha}=\{0\}$. If $r_{\alpha} I_{\beta} \neq\{0\}$, then there exists $s_{\beta} \in I_{\beta}$ with $r_{\alpha} s_{\beta} \neq 0$, so $0 \neq\left(a+s_{\beta}\right) r_{\alpha}=s_{\beta} r_{\alpha} \in I$ which implies that $a \in h(P)$. Thus we may assume that $r_{\alpha} I_{\beta}=\{0\}$. Since $I_{0} I_{\alpha} \neq\{0\}$, let $c \in I_{0}, d \in I_{\alpha}$ with $c d \neq 0$. If $\beta=0$, then $0 \neq$ $(a+c)\left(r_{\alpha}+d\right)=c d \in I_{0}$ implies that $a \in h(P)$. If $\beta=1$, then $0 \neq\left(a^{2}+c\right)\left(r_{\alpha}+d\right)=c d \in I_{0}$ implies that $a^{2} \in h(P)$ and hence $a \in h(P)$, since $P$ is a prime.

Now we generalize Proposition 4.2.

Theorem 4.3. Let $G$ be a finite abelian group of order $n$, and let $R$ be a commutative graded super-ring. Suppose I is a P-weakly primal graded superideal of $R$ with $\left(I_{e}^{0}\right)\left(I_{g}^{\alpha}\right) \neq\{0\}$ for all $g \in G$ and $\alpha \in \mathbb{Z}_{2}$. If $P$ is a prime graded superideal of $R$ then I is $P$-primal.

Proof. It is enough to show that $v(I)=h(P)$. If $a \in h(P)$, then $a$ is not prime to $I$. So, $a \in v(I)$. Assume that $a \in v(I)$. We show that $a \in h(P)$. Suppose $a \in R_{x}^{\beta}$ where $x \in G$ and $\beta \in \mathbb{Z}_{2}$. Then there exists $r_{g}^{\alpha} \in h(R)-h(I)$ with $r_{g}^{\alpha} a \in I$. If $r_{g}^{\alpha} a \neq 0$, then $a \in h(P)$ since $I$ is a $P$-weakly primal graded superideal of $R$. Therefore we may assume that $r_{g}^{\alpha} a=0$. If $a I_{g}^{\alpha} \neq\{0\}$, then there exists $r^{\prime} \in I_{g}^{\alpha}$ with $a r^{\prime} \neq 0$. Thus, $0 \neq a\left(r_{g}^{\alpha}+r^{\prime}\right) \in I$ and $r_{g}^{\alpha}+r^{\prime} \notin I$; hence $a \in h(P)$. Therefore we may assume that $a I_{g}^{\alpha}=\{0\}$. Let $m$ be the smallest positive integer with $x^{m}=e$, where $e$ is the identity element in $G$. Then $a^{2 m} \in R_{e}^{0}$, since $2 m \beta=0 \in \mathbb{Z}_{2}$. Now, if $r_{g}^{\alpha} I_{e}^{0} \neq\{0\}$, then there exists $s \in I_{e}^{0}$ with $r_{g}^{\alpha} s \neq 0$. So, $0 \neq\left(a^{2 m}+s\right) r_{g}^{\alpha} \in I$ which implies that $a^{2 m} \in P$ and hence $a \in h(P)$, since $P$ is a prime graded superideal of $R$. Therefore we may assume that $r_{g}^{\alpha} I_{e}^{0}=\{0\}$. Since $\left(I_{e}^{0}\right)\left(I_{g}^{\alpha}\right) \neq\{0\}$, there exists $c \in I_{e}^{0}$ and $d \in I_{g}^{\alpha}$ with $c d \neq 0$. Now, $0 \neq c d=\left(a^{2 m}+c\right)\left(r_{g}^{\alpha}+d\right) \in I_{g}^{\alpha}$ and $r_{g}^{\alpha}+d \notin I_{g}^{\alpha}$ implies that $a^{2 m}+c \in P_{e}^{0}$. So, $a^{2 m} \in P_{e}^{0} \subseteq h(P)$; hence $a \in h(P)$.

Let $G$ be a group of order $n$. We recall that if $P$ is a prime graded superideal of $R$ and $I$ is a $P$-weakly primal graded superideal of $R$ but not $P$-primal, then, by Theorem 4.3 , there exists 
$g \in G$ and $\alpha \in \mathbb{Z}_{2}$ such that $\left(I_{e}^{0}\right)\left(I_{g}^{\alpha}\right)=\{0\}$. Therefore if $g=e$ and $\alpha=0$, then $\left(I_{e}^{0}\right)^{2}=\{0\}$. Hence, by Theorem 2.13(1), $I^{4 n}=\{0\}$ which implies that $\sqrt{I}=\sqrt{\{0\}}$.

Definition 4.4. Let $I$ be a graded superideal of $R$, and let $P$ be the smallest graded superideal of $R$ satisfying the following condition: if $r, a \in h(R)$ with $r a \in I$, then $r \in I$ or $a \in P$. Then, $I$ is called a $P$-primary graded superideal of $R$.

\section{Remark 4.5.}

(1) If $I$ is a $P$-primary graded superideal of $R$, then $\sqrt{I} \subseteq P$.

(2) $I$ is a primary graded superideal of $R$ if and only if $\sqrt{I}=P$.

Now we have the following result about primary graded superideals.

Proposition 4.6. Every primary graded superideal of $R$ is primal.

Proof. Let $I$ be a primary graded superideal of $R$. We prove that $\rho(I)=h(\sqrt{I})$.

Let $r \in \rho(I)$, then there exists $a \in h(R)-h(I)$ with $r a \in I$. Because $I$ is a primary graded superideal of $R$, then $r \in h(\sqrt{I})$.

Conversely, assume that $b \in h(\sqrt{I})$. If $b \in I$, then $b$ is not prime to $I$; hence $b \in \rho(I)$. So, we may assume that $b \notin I$. Let $n$ be the smallest positive integer such that $b^{n} \in I$. Then $n \geq 2$ and $b^{n-1} \notin I$. Now, $b^{n}=b b^{n-1} \in I$ and $b^{n-1} \notin I$ implies that $b \in \rho(I)$. Thus, $\rho(I)=h(\sqrt{I})$.

The next result is a generalization of [6, Theorem 1] to the graded supercase.

Proposition 4.7. Every irreducible graded superideal of $R$ is primal.

Proof. Let $I$ be an irreducible graded superideal of $R$ and let $b_{1}, b_{2} \in h(R)$ be not prime to $I$. Then $I \varsubsetneqq\left(I: b_{1}\right)$ and $I \varsubsetneqq\left(I: b_{2}\right)$. Hence

$$
I \varsubsetneqq\left(I: b_{1}\right) \cap\left(I: b_{2}\right)=\left(I:<b_{1}>+<b_{2}>\right),
$$

since $I$ is irreducible, where $\langle a\rangle=a R$ for $a \in h(R)$. One can easily check that

$$
\left(I:<b_{1}>+<b_{2}>\right)=\left(I:<b_{1}>+<b_{1}-b_{2}>\right),
$$

which implies that

$$
I \varsubsetneqq\left(I: b_{1}\right) \cap\left(I: b_{1}-b_{2}\right) .
$$

Therefore, $I \varsubsetneqq\left(I: b_{1}-b_{2}\right)$; hence $b_{1}-b_{2}$ cannot be prime to $I$. Moreover, $I \varsubsetneqq\left(I: b_{1}\right)$ implies that $r b_{1}$ cannot be prime to $I$ for any $r \in h(R)$. So, $I$ is a primal graded superideal of $R$.

Recall that $R$ is a graded superdomain if $R$ is a commutative graded super-ring with unity and $h(R)$ has no divisors of zero. 
Proposition 4.8. A graded superideal of a graded superdomain is primal if and only if it is weakly primal.

Proof. We may assume that $I \neq\{0\}$. Suppose that $I$ is a $P$-primal graded superideal of $R$. We show that $I$ is a weakly $P$-primal graded superideal of $R$. Let $a \in h(P)-\{0\}$. If $a \in I$ then $a$ is not weakly prime to $I$. So we may assume that $a \notin I$. Because $I$ is $P$-primal, there exists $b \in h(R)-h(I)$ with $0 \neq a b \in I$ since $R$ is a graded superdomain. Thus $a$ is not weakly prime to $I$. On the other hand, if $a$ is not weakly prime to $I$ then $a \neq 0$ and $a$ is not prime to $I$ since $R$ is a graded superdomain. Thus, $a \in h(P)-\{0\}$.

Conversely, suppose that $I$ is a weakly $P$-primal graded superideal of $R$. We show that $I$ is $P$-primal. By Proposition 2.4, $P$ is a weakly prime graded superideal of $R$. Hence it is a prime graded superideal of $R$, since $R$ is a graded superdomain. It is enough to show that $h(P)$ is exactly the set of elements of $h(R)$ that are not prime to $I$. Clearly, $0 \in h(P)$. Let $0 \neq a \in h(P)$. Then $a$ is not weakly prime to $I$. So it is not prime to $I$. On the other hand, suppose that $a \in h(R)$ is not prime to $I$. If $a=0$ then $a \in h(P)$, so we may assume that $a \neq 0$. Then there exists $r \in h(R)-h(I)$ with $r a \in I$. Since $R$ a graded superdomain we have that $0 \neq a r \in I$. So $a$ is not weakly prime to $I$; hence $a \in h(P)$.

By using Proposition 4.6, Proposition 4.7 and Proposition 4.8, we have the following result.

Corollary 4.9. Let $R$ be a graded superdomain. Then the following hold.

(1) Every primary graded superideal of $R$ is weakly primal.

(2) Every irreducible graded superideal of $R$ is weakly primal.

\section{References}

[1] D. Anderson and E. Smith, Weakly prime ideals, Houston, J. Math. 29(2008), 831-840.

[2] S. E. Atani, On graded prime submodules, Chaing Mai J. Sci., 33(1)(2006), 3-7.

[3] S. E. Atani, On graded weakly prime ideals, Turkish Journal of Mathematics, 30(2006), 351-358.

[4] Y. A. Bahturin, A. Giambruno, Group gradings on associative algebras with involution, DOI:10.4153/CMB2008-020-7, Canad. Math. Bull., 51(2008), 182-194.

[5] M. Behboodi, H. Koohi, Weakly prime submodules, Vietnam J. Math., 32(2)(2004), 185-195.

[6] L. Fuchs, On primal ideals, Amer. Math. Soc. 1(1950), 1-6.

[7] M. E. Moore, S. J. Smith, Prime and radical submodules of modules over commutative rings, Comm. Algebra, 30(10)(2002), 5037-5064.

[8] P. F. Smith, Primary modules over commutative rings, Glasgow Math. J., 43(2001), 103-111.

Department of Mathematics, The Hashemite University, Zarqa 13115, Jordan.

E-mail: ameerj@hu.edu.jo 